\title{
Algoritmos genéticos como herramienta de decisión frente a diversos escenarios de pronóstico hidrológico
}

Genetic algorithms as decision making tools for hydrological forecast in diverse scenarios

Facundo Alonso ${ }^{1}$, Juan Bertoni ${ }^{1}$, César García ${ }^{2}$ y Carlos García ${ }^{3}$

\section{RESUMEN}

Los modelos de pronóstico hidrológico son herramientas matemáticas que se emplean mientras se está produciendo una tormenta con el fin de predecir el caudal que se producirá en una sección de un río con un cierto tiempo de anticipación (horizonte de pronóstico). Como información de entrada suelen utilizar la lluvia y el caudal de la sección de interés, registrados en tiempo real. Sin embargo, durante un evento de gran magnitud, algunos sistemas de pronóstico experimentan una degradación en la calidad de los caudales observados en tiempo real, y en algunos se produce pérdida de dicha información. Esta circunstancia lo vuelve un simple modelo de simulación. El juego de valores de parámetros que mejor se ajusta al modelo en modo pronóstico, no es necesariamente el óptimo para el mismo modelo en modo simulación, es decir, sin información de caudal observado en tiempo real. En este trabajo se emplea el método de algoritmos genéticos MOCOM-UA para realizar una optimización multiobjetivo del modelo hidrológico GR4P. El objetivo es ofrecer al operador del sistema de alerta de crecidas una herramienta que facilite la toma de decisión ante escenarios diversos. El método se basa en un frente de Pareto determinado por un conjunto de soluciones de compromiso entre dos escenarios a optimizar, que están dados por la mejor performance del modelo matemático frente a: 1) con la disponibilidad de caudales a tiempo real, y 2) sin dicha disponibilidad. La metodología se aplicó en el río Moros en la Bretaña francesa. Los resultados indican que la solución óptima para un escenario produce escasa performance en el otro, ya que se aparta del juego de valores de parámetros para el cual fue calibrado el modelo. Por otro lado, las soluciones de compromiso ofrecen performances aceptables para ambos objetivos. Este estudio es parte del proyecto HYRADIER, una cooperación científica entre Argentina, Brasil y Francia, financiado por el programa STIC-AmSud.

Palabras clave: algoritmos genéticos; horizontes de pronóstico; modelos hidrológicos.

\section{ABSTRACT}

Hydrological forecasting models are mathematical tools that can be used, while a storm is happening, to predict the state of a river flow in a future time (forecasting horizon). Input data usually consist of rainfall and river flow information of the study area, reg-

\footnotetext{
1 Universidad Nacional de Córdoba. Argentina.

2 Concejo Nacional de Investigaciones Científicas y Técnicas. Córdoba, Argentina.

3 Universidad Católica de Córdoba. Argentina.
} 
istered at real time. However, during high magnitude events, some forecasting systems experiment degradation in the quality of the real time flow data and sometimes even data loss. In these cases the model becomes a simulation model. The set of parameters that produce a good performance in a forecasting model are not necessarily the optimum combination for the same model in a simulation mode (i.e. without real time flow measurement). This paper explores the MOCOM-UA genetic algorithm as a method to perform a multiobjective optimization of the GR4P hydrological model. The goal is to provide a tool for an early warning flood system operator to facilitate decision making under different scenarios. The method is based in a Pareto front determined by a set of compromise solutions between two scenarios to be optimized, which are the best performance of the mathematical model with: 1) real time flow measurements and 2) when these measurements are not available. The method was applied and tested in the Moros river basin in the French Brittany region. Results indicate that optimal parameters for a scenario produce poor performance in the other. On the other hand, compromise solutions produced a good performance for both objectives. This study is part of the HYRADIER project, a scientific cooperation of Argentina, Brazil and France, financed by the STIC-AmSud project.

Keywords: genetic algorithms; forecasting horizons; hydrological models.

\section{ICHIKLLACHAW}

Idruluhiku imanaw shamuq watakunachaw kananta rikaynapaqmi yupaykunawan ruraykuna kayan. Atska tamya kaptin imaykama mayu wiñananta riqinapaqmi allí tsay mudilukuna. Nawpa willakuyqa tamyawan imayka mayu kankanllam. Sitsun atska tamya kaptinqa, manam mayupa yakun tsaynawllanatsu, atskayanmi. Kaynaw kaptinqa manan kikin willakuyllanatsu, tsayqa tumarin, tikrarin huklaayallamannam. Itsapis llapan kikin willakuyta riqintsiknatsu, tsay ichik willakuykuna uqrakan. Kaynaw kaptinqa manan huk ashpu mudilullanatsu, sinuqa hatun sasayashqa mudilunam. Kay uryachaw ñawisakashqa MOCOM-UA ginitiku alguritmu mitudu nishqanmi allinpa rikakaananpaq GR4P idrulugiku mudilu nishqanta. Kay rurayqa ashin allin naanita akranapaq tukuy rikuq kaqkunachaw. Kay mituduqa rurakashqa paritupa allí kaq quchuntin shumaq ruraykunachawmi ishkay patsa alliyaatsinapaq yupay mudiluwan: 1) Kawdal kanqanchaw huk patsachaw, niykur 2) mana kawdal kanqanchaw. Kay mituduqa rurakashqa Murus mayuchawmi, francesa Bretaañachaw. Kay urya ushan, allikaq isinariyu tikratsin ichik huk kaqpa pirurmansinman. Hananman, nin alli ruraykuna ishkantam shumaq pushan. Kay uryaqa ichikllan kaqmi, hatun HYRADIER pruyiktupa, kay rurakan yanapanakuypa Argintina, Brasil niykur Francia, STIC-AmSud Programapa qillayninwanmi.

Pushaq shimikuna: ginituku alguritmu; shamuqpaq willakuy; hidrulugiku mudilukuna.

\section{INTRODUCCIÓN}

La hidrología aplicada posee dos objetivos principales. El primero, consiste en la predeterminación de ciertos caudales de crecida o estiaje con el propósito de dimensionar obras (presas, vertederos, puentes, etc.). Esta evaluación cuantitativa no está asociada a un evento en particular, sino que se busca evaluar la probabilidad de superar una determinada marca de crecida, en términos por ejemplo, del periodo de retorno. Este tipo de análisis es esencialmente orientado en tiempo diferido, a través de la simulación/ 
reconstrucción de eventos pasados a la luz de las observaciones hidrometeorológicas históricas. Por el contrario, el otro objetivo de la modelización hidrológica corresponde al pronóstico de crecidas o sequías. En este caso, se busca evaluar la probabilidad de superar un umbral de caudal frente a un horizonte determinado de antelación (desde algunos minutos a algunos días, dependiendo del tiempo de respuesta del sistema hidrológico a las fueras meteorológicas actuantes), es decir, realizar el pronóstico a tiempo real.

Para maximizar la calidad del pronóstico en el horizonte elegido, es deseable partir del mejor estado posible en el instante de la previsión, y es por lo tanto conveniente «re-ajustar» el modelo, si es posible, sobre la base de los últimos caudales observados a lo largo de algunos pasos de tiempo precedentes al instante en el cual debe ser emitido el pronóstico. Este procedimiento es descripto esquemáticamente en la figura 1.

A causa de esta etapa, denominada de asimilación (que corrige el estado del modelo), un buen modelo de pronóstico no es necesariamente un buen modelo de simulación, y viceversa. Debido al elevado peso asignado a las últimas observaciones, la estructura de un modelo hidrológico de pronóstico puede típicamente ser más simple que aquella de un modelo de simulación resultando potencialmente bastante desconcertante tanto por los desarrolladores como por los utilizadores de estos modelos (Berthet, 2010).

Sin embargo, desde el punto de vista del desarrollo de los modelos hidrológicos, la confrontación entre estos dos objetivos (simulación y pronóstico) no es necesariamente tan clara como parece. En efecto, como lo recuerda Duband (1999), para una utilización operacional en pronóstico, se necesita de una modelización específica notoriamente distinta a las necesidades de la hidrología aplicada orientada a la comprensión y a la reconstrucción del ciclo del agua: simular no es pronosticar. De todos modos, el progreso en el conocimiento puede contribuir tanto a uno como al otro objetivo. En efecto, para el caso de un modelo de pronóstico hidrológico, altamente dependiente de la actualización de estado a cada paso de tiempo, si la cadena de transmisión de datos hidrometeorológicos (red pluviométrica e hidrométrica) que alimenta el sistema de pronóstico falla, las performances reales pueden volverse muy por debajo de aquella que uno esperaría en un caso ideal.

En particular, si la información a tiempo real de los últimos datos de caudales observados son perdidos (daño en la estación hidrométrica, en la red de comunicación, etc.) y no puede ser más asimilado, el modelo hidrológico se vuelve en una situación típica de simulación, ejercicio para el cual otro modelo (o el mismo modelo, pero parametrizado distinto) podría dar resultados más confiables.

El especial interés de los datos de radar para el pronóstico hidrológico es debido a la posibilidad de contar con información más fina que la proveniente de la red telemétrica de sensores pluviográficos sobre la distribución espacial de las precipitaciones así como sobre las velocidades y direcciones de desplazamiento de las células de lluvia.

Esto es particularmente notorio cuando el pronóstico y la alerta temprana deben ser hechos para cuencas hidrográficas de área entre pequeña y mediana, con tiempos de respuesta inferiores a 6-8 hs., máxime para zonas fuertemente urbanizadas. Sin embargo, el radar debe ser calibrado empleando datos pluviométricos locales, siendo por lo tanto dependiente de la calidad de la información pluviométrica existente en los sitios de aplicación del pronóstico. 


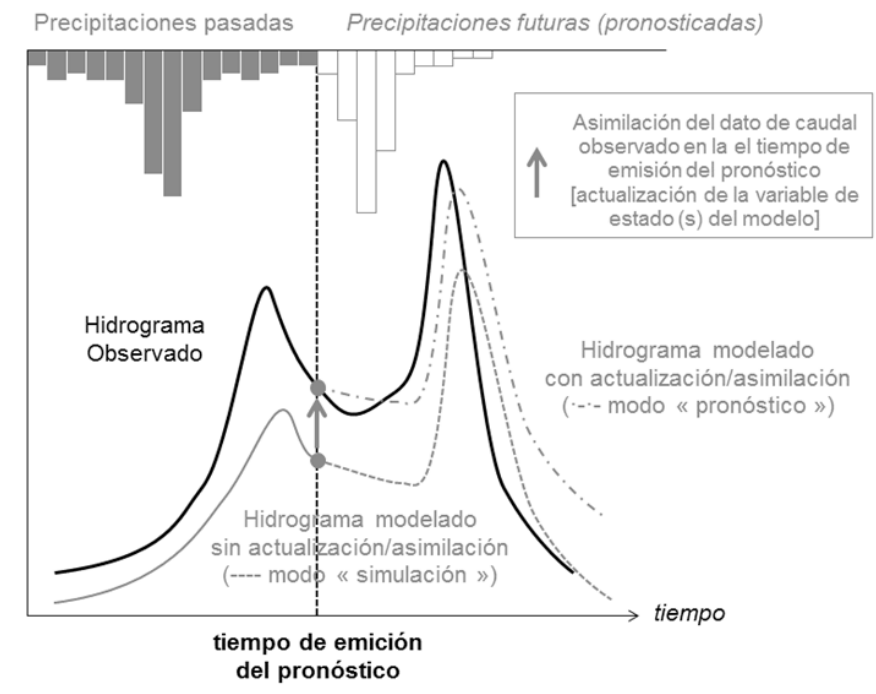

Figura 1. Hidrograma simulado y pronosticado

El estudio de incertidumbre asociada al acople radar-modelización hidrológica y la evaluación de los riesgos de falsa alarma pueden aportar una contribución importante para la concepción y la gestión de los sistemas de alerta temprana de crecidas.

En este trabajo, que forma parte del proyecto HYRADIER, una cooperación científica entre Argentina, Brasil y Francia, financiado por el programa STIC-AmSud, se propone una metodología binaria simulación/pronóstico, como enfoque de una visión más matizada y continua entre las situaciones de pronóstico en contexto bien instrumentado, donde la información a tiempo real puede ser plenamente puesta al servicio de la asimilación, y poco instrumentado, donde el modelo está levemente restringido ya que carece de información de caudal a tiempo real, dejado por lo tanto libre de evolucionar en condiciones próximas a las de un modelo de simulación.

En particular, se pretende saber si es siempre preferible calibrar un modelo de pronóstico utilizando específicamente los criterios (parámetros) de pronóstico y en modo pronóstico, o si puede ser interesante dar un peso a los criterios más cercanos a un enfoque de simulación. El estudio de la determinación de la combinación óptima, que dependerá eventualmente de los contextos fisiográficos y meteorológicos, fue efectuado en este trabajo con series de datos sobre la cuenca del río Moros en su desembocadura en Concarneau, región de la Bretaña al noroeste de Francia.

\section{MATERIALES Y MÉTODOS}

En cuanto a las herramientas de modelación, los trabajos se apoyaron sobre los modelos desarrollados por el equipo de hidrología del Irstea (ex Cemagref) de Antony, Francia: el modelo GR4J (Perrin, 2003) para la simulación y el modelo GRP (Tangara, 2005; Berthet, 2010) para el pronóstico. Estos modelos, que funcionan a paso de tiempo diario u horario, fueron probados sobre un gran número de cuencas hidrográficas en Francia, Estados Unidos, Australia, Brasil y México. El modelo GRP es en particular utilizado por varios servicios operacionales de pronóstico de crecidas en Francia. En este trabajo se emplea un modelo que denominaremos GR4P, que puede ser interpretado como una combinación de los anteriores. El estudio de sensibilidad de las performances de los modelos frente a los diversos criterios de calibración de los parámetros fue efectuado con la ayuda del método de optimización global multiobjetivo MOCOM-UA (Yapo, 1998). 
Modelo Lluvia-Caudal

El modelo GR4P resulta de la combinación de los modelos GR4J y GRP. El modelo GR4J presenta la estructura que se muestra en la figura 1. Para una descripción detallada del mismo, remitirse a (Perrin, 2003). A los fines de este artículo vasta con destacar que se trata de un modelo conceptual lluvia-caudal (PQ) de reservorios, concentrado, de simulación continua. El mismo dispone de un bloque de producción (enfocado en la estimación del volumen escurrido) y otro de transferencia (enfocado en la distribución temporal del mismo). Las variables de entrada son la Precipitación (P) y la Evapotranspiración Potencial (E). Luego de calculados los valores netos (Pn y En), se estima la porción de lluvia que se infiltra (Ps) y la que escurre directamente (Pr); o bien la porción evaporada de la superficies del suelo (Es). El agua que infiltra, llena un reservorio de producción, cuya variable de estado es $S k$, y cuya máxima capacidad es el parámetro $\mathrm{x}_{1}$. Una porción de agua percola (Perc) y se une a Pr conformando la porción de que escurre sobre la superficie hasta el punto de concentración de la cuenca. El agua es transitada empleando dos herramientas: hidrogramas unitarios (HU1 y HU2), cuyo tiempo base es función del parámetro $\mathrm{x}_{4}$; $\mathrm{y}$ de un reservorio de transferencia que modela el almacenamiento sobre superficie del agua en la cuenca, cuya variable de estado es $R k$, y su máximo valor el parámetro $\mathrm{x}_{3}$. Una función del parámetro $\mathrm{x}_{2}$, redistribuye el agua de escurrimiento directo.

El modelo GRP, resulta de transformar el modelo GR4J al modo pronóstico y llevarlo a su forma más simplificada (estructura sencilla y solo tres parámetros). Una descripción detallada del modelo GRP puede leerse en Berthet (2010). Dado que se trata de un modelo de pronóstico, fue diseñado considerando que se dispone en cada pdt el valor del caudal observado a tiempo real. Partiendo de esta consideración fue agregado a su estructura un algoritmo que actualiza (re-ajusta) el estado Rk del sistema bajo la premisa de que el caudal simulado en el pdt anterior al estado actual del sistema debe ser el valor preciso para generar el caudal observado en el instante actual, mejorando de ese modo la estimación del caudal para el pdt siguiente.

Finalmente, el modelo GR4P, es una versión completa del modelo GR4J (es decir, sin las simplificaciones realizadas para el GRP), pero conservando el algoritmo de actualización la que dispone el modelo GRP. Otro componente adicional del modelo GR4P es su facultad de simular series de tiempo con pdt diario y horario.

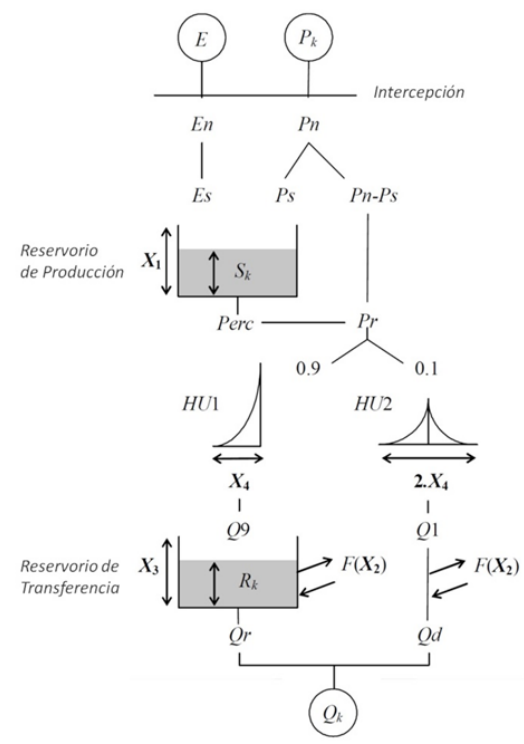

Figura 2. Estructura del modelo GR4J (Perrin y Adréassian, 2003) 


\section{Método de Optimización}

Las investigaciones en métodos de optimización han conducido al uso de estrategias de búsqueda basadas en hacer evolucionar una población (Brazil, 1987, 1988; Wang, 1991; Duan, 1992, 1993; Sorooshian. 1993, entre otros). En este sentido, el algoritmo de optimización global mono-objetivo Shuffled Complex Evolution (SCE-UA) ha demostrado ser consistente, efectivo y eficiente en la localización del juego de parámetros del modelo hidrológico óptimo globalmente con respecto a alguna función objetivo dada (Duan, 1992, 1993; Sorooshian, 1993; Luce, 1994; Gan, 1996; Tanakamaru, 1995, 1996, entre otros). En este trabajo se emplea el método de optimización global MOCOM-UA que es la versión multi-objetivo del método SCE-UA. El MOCOM-UA, se encuentra descripto en detalle en Yapo (1998). Este método parte precisamente de una población inicial con $\mathrm{N}$ individuos, generados con distribución probabilística uniforme dentro del hiperespacio de validez de los parámetros, cada uno de los cuales representa un juego de valores de parámetros del modelo a optimizar. A lo largo de un conjunto de pasos basados en las estrategias de búsqueda de los algoritmos genéticos, la población evoluciona hacia nuevos valores de parámetros que tienden a satisfacer simultáneamente todas las funciones objetivo (FO). De este modo se procura alcanzar el frente de Pareto, dado por el conjunto de individuos (juegos de valores de parámetros) no dominados (es decir, ningún individuo es mejor que otro en todas las FO) que representan soluciones de compromiso entre las soluciones óptimas extremas. Para el caso de realizar una optimización entre dos funciones objetivo (FO1 y FO2), el frente de Pareto tendrá un individuo que representa el mejor valor de la FO1, otro que representa el mejor valor de la FO2, y un conjunto de soluciones de compromiso entre ambas soluciones extremas.

Funciones objetivo y criterio de evaluación del ajuste

La expresión matemática adoptada como FO para calibrar el modelo y también para evaluar su performance fue el coeficiente KGE (Gupta, 2009) descripto en la Ecuación (1).

$$
K G E=1-\sqrt{(r-1)^{2}+(\alpha-1)^{2}+(\beta-1)^{2}}
$$

Donde r es el coeficiente de correlación lineal entre Qobs y Qsim, a es una medida de variabilidad en los valores de los datos (igual a la relación entre el Desvío Estándar de Qsim sobre el Desvío Estándar de Qobs), y b es igual a la relación entre el promedio de Q sim sobre el promedio de Qobs. Este criterio de ajuste toma valores desde - $\infty$ a 1.

El trabajo se plantea en el contexto de los dos escenarios factibles durante el proceso operativo de pronóstico:

Escenario 1: el sistema de transmisión de datos de Qobs a tiempo real funciona adecuadamente, por lo que el operador del modelo de pronóstico cuenta con la posibilidad de actualizar el estado del modelo en cada paso de tiempo a partir del valor conocido del caudal observado.

Escenario 2: el sistema es incapaz de enviar la información de caudal a tiempo real, por lo que el modelo funciona como un simple modelo de simulación, ya que no puede actualizar su estado. El operador debe pronosticar con el modelo en esa situación.

A partir de estos dos escenarios, se definen las FO de la siguiente manera: FO1: KGE para la calibración del modelo funcionando en condiciones del escenario 1 (sin Qobs a tiempo real). FO2: KGE para la calibración del modelo funcionando en condiciones del escenario 2 (con Qobs a tiempo real). 


\section{Cuenca Hidrográfica y Datos}

El presente trabajo se enmarca en el proyecto tri-nacional Hyradier, financiado por el Programa STIC-AMSUD (2015), el cual tiene por objetivo establecer conclusiones comparativas entre cuencas hidrográficas de los países relacionados (Argentina, Francia y Brasil), con tiempos de reacción semejantes. En este contexto, se eligió como primera fase de aplicación la cuenca hidrográfica francesa «Moros en Concarneau» ubicada en la región de Bretaña, al noroeste de Francia. Cuenta con un tiempo de reacción aproximado de 7,7 hs., un área de $20.97 \mathrm{~km}^{2}$, y dispone de una serie de 35060 datos a paso de tiempo (pdt) horario (01/08/2009 al 01/08/2013). La metodología aplicada para evaluar la performance del modelo PQ fue un método de selección de muestras de prueba (Oudin, 2005) tal como lo recomienda Klemes (1986): se divide en dos periodos de calibración/control, en este caso de 22030 pdt cada uno; y se adopta un periodo de inicialización, en este caso 9000 pdt. Luego, la cantidad de datos de cada sub-periodo, viene de considerar la expresión siguiente:

$[($ Serie completa - Inicialización $) / 2]+$ Inicialización $=[(35060-9000) / 2]+9000=22030$

Donde:

Periodo $1=01 / 08 / 2009$ al 04/02/2012 (con 9000 pdt para inicializar)

Periodo $2=27 / 01 / 2011$ al 01/08/2013 (con 9000 pdt para inicializar)

Luego, el modelo es calibrado en cada sub-periodo, y probado en el otro.

\section{Optimizacion del modelo GR4P}

Se realizó la optimización del modelo hidrológico GR4P para horizontes de previsión $\mathrm{L}=1, \mathrm{~L}=6, \mathrm{y} \mathrm{L}=24$ horas, como se muestran en la figura 3, tomando el primer periodo de datos que fue definido previamente. A los fines de provocar un ahorro de consumo computacional, se permitió al método de optimización concluir el proceso antes de alcanzar el frente de Pareto final, a condición de obtener al menos el 85\% de los 60 individuos de la población no-dominados, y una tolerancia al cambio del valor de FO de 0,001 entre dos generaciones poblacionales. Por esta razón, se observa en la gráfica que no todos los individuos de la población optimizada son no-dominados.

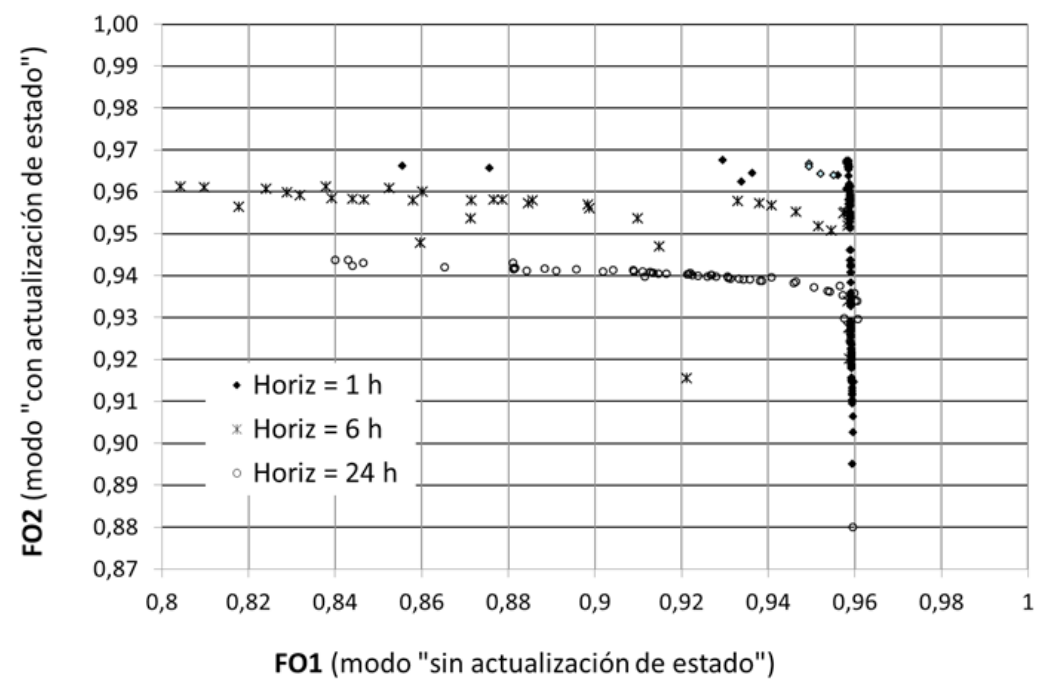

Figura 3.Optimización del Modelo GR4P para distintos horizontes de pronóstico 
Para los tres horizontes de pronóstico (L) se llega al mismo «mejor valor» de FO1. En cambio, el mejor valor de FO2, se degrada cuanto mayor es el valor de L. Por otra parte, para $\mathrm{L}=1 \mathrm{~h}$, el «mejor valor de FO2» es mayor que el «mejor valor de FO1», por lo que la actualización de estado mejora la performance; para $\mathrm{L}=6 \mathrm{~h}$, la performance es indiferente a la actualización de estado; y para $\mathrm{L}=24 \mathrm{~h}$, parece que la actualización de estado desmejora la performance. Es decir, hay una notoria tendencia de desmejorar la performance debido a la actualización de estado, a medida que aumenta el horizonte L. Esto puede ser explicado porque el procedimiento de actualización del modelo, obliga a que a cada paso de tiempo el valor pronosticado tome exactamente el valor observado en cada Dt, quitándole de este modo grados de libertad en la selección del juego óptimo de parámetros.

Con la población optimizada, se realizó el control de la calidad de la calibración, tomando el segundo periodo de datos de la cuenca definido previamente. En la figura 4 se muestran 30 individuos representativos de la población optimizada, donde se observa el valor del coeficiente KGE para cada uno en la fase de calibración (primer periodo de datos), y control (segundo periodo), tanto para el modelo «sin actualización» (escenario 1), y «con actualización» (escenario 2), para un horizonte de pronóstico (L) de 1 hora.

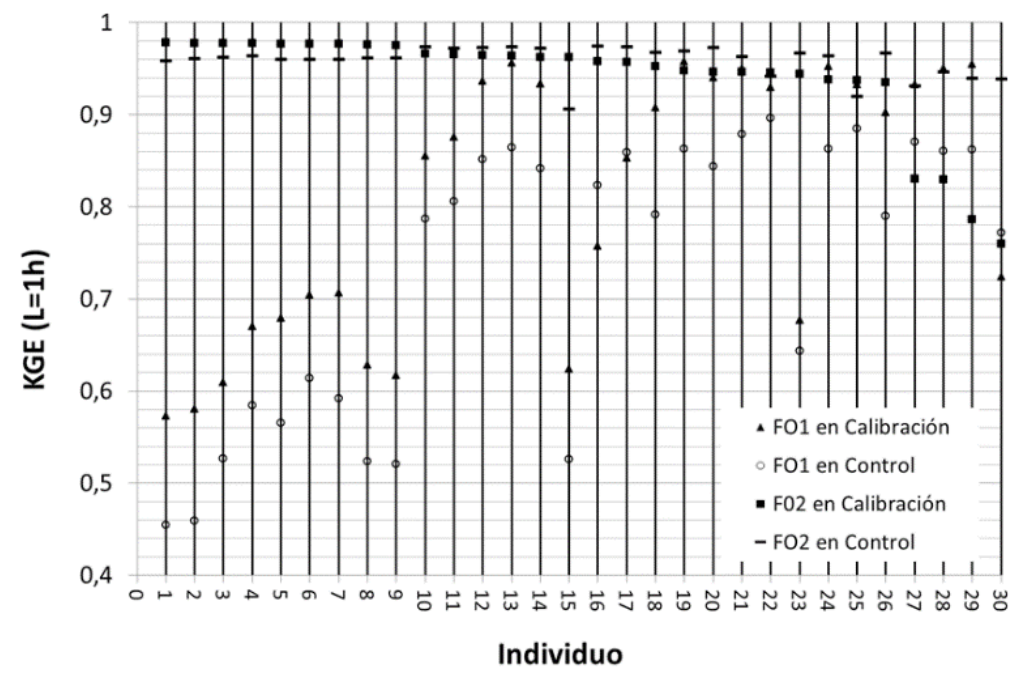

Figura 4. Performance de la población optimizada para los distintos escenarios

Los individuos son ordenados de mejor a peor valor de la FO2 en calibración. Esto quiere decir que el individuo 1 tiene el mejor valor de la FO2 en calibración, y el individuo «30», el peor valor. Se observa que, si bien la población no arribó al frente de Pareto final, presenta individuos con buena performance tanto en calibración como en control, lo cual demuestra que hay ciertas combinaciones de parámetros robustas ya que son capaces de adaptarse con buena performance a cualquiera de los escenarios. Se puede ver también que los valores de las FO1 mejoran al mismo tiempo que los valores de la FO2 se degradan. Finalmente, se puede ver que los puntos que tienen valores de la FO1 buenos en calibración, tienen también buenos valores en control.

En la figura 5 se muestran en puntos la performance de la población óptima calibrada en el primer periodo de la serie de datos, y en cruces las performances de las simulaciones de los mismos individuos en el segundo periodo de la serie (control). 
Como se reflexionó previamente, se puede identificar puntos buenos para ambos periodos (punto 22, por ejemplo). Realizando un cambio de escala de los ejes del gráfico, se puede mirar con mejor detalle en la figura 5 y 6 y en la figura 7 la posición del individuo 22 y sus valores del coeficiente de KGE para cada escenario explicado.

Si bien el punto «22» tiene buen valor para las FO1 y FO2, hay puntos que pueden ser mejores en la FO1 en calibración y en control, y otros puntos que son mejores en FO2 también en calibración y en control. Por ejemplo, el punto «4» tiene mejor valor que el punto «22» tanto en calibración como en control para la FO2. Pero en FO1 es inferior.

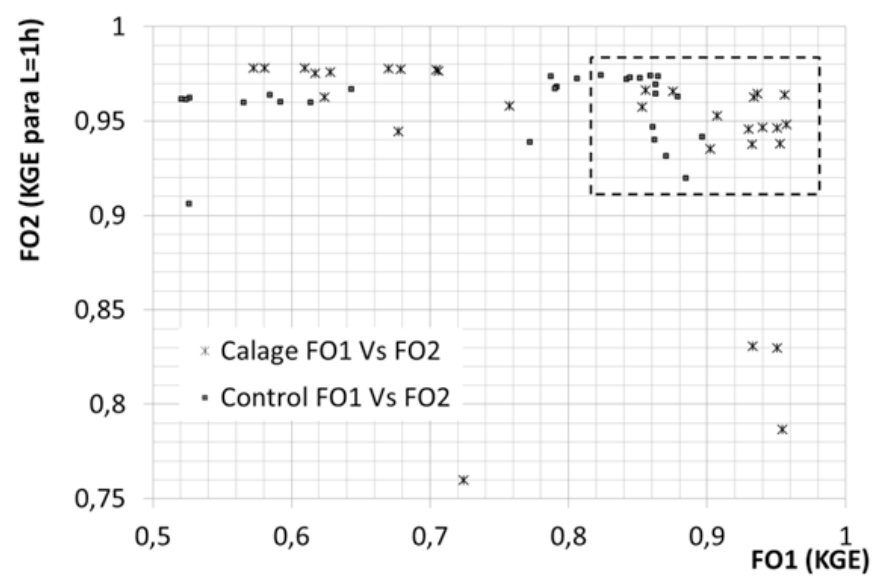

Figura 5. Población optimizada para $\mathrm{L}=1 \mathrm{~h}$

Ahora bien, la mejora en FO2 que tiene el punto 4 respecto del punto 22, justifica abandonar la ventaja de contar con un único juego de valores de parámetros que interprete al modelo. En efecto, al adoptar dos soluciones buenas (una para FO1 y otra para FO2), se requiere en modo operativo dos corridas en paralelo del modelo pero calibrado con dos juegos de parámetros distintos.

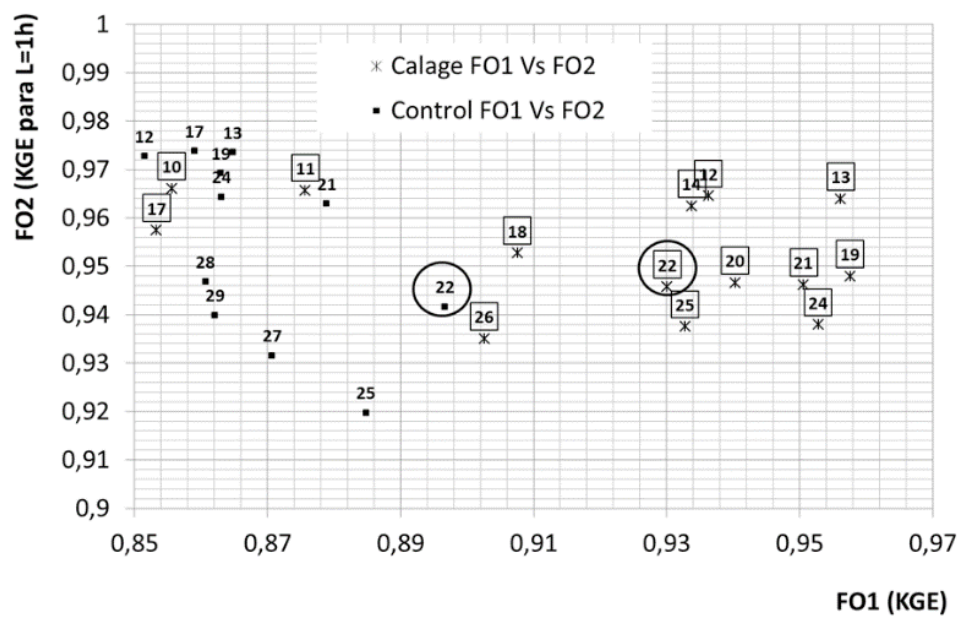

Figura 6. Detalle de la población optimizada para $\mathrm{L}=1 \mathrm{~h}$

En la tabla 1 se muestran los valores de los parámetros correspondientes al punto 4, y al punto 22, en comparación con los valores de los parámetros optimizados por el IRSTEA para la cuenca en estudio. 
Tabla 1. Performance del modelo para diversos escenarios.

\begin{tabular}{|c|c|c|c|c|c|c|c|c|c|c|c|}
\hline \multirow{3}{*}{ Posición } & \multicolumn{4}{|c|}{ Parámetros } & \multicolumn{2}{|c|}{ Calibración } & \multicolumn{5}{|c|}{ Control } \\
\hline & \multirow[t]{2}{*}{$\mathrm{X} 1$} & \multirow[t]{2}{*}{$x 2$} & \multirow[t]{2}{*}{ X3 } & \multirow[t]{2}{*}{$\mathrm{X} 4$} & \multirow[t]{2}{*}{$\begin{array}{c}\text { KGE } \\
\text { sin actualiz }\end{array}$} & \multirow[t]{2}{*}{$\begin{array}{c}\text { KGE } \\
\text { con act. } \\
(1 \mathrm{~h})\end{array}$} & \multirow[t]{2}{*}{$\begin{array}{c}\text { KGE } \\
\text { sin act. }\end{array}$} & $\begin{array}{c}\text { KGE } \\
\text { con act. } \\
\text { L (h) }\end{array}$ & $\begin{array}{c}\text { KGE } \\
\text { con act. } \\
\text { L (h) }\end{array}$ & $\begin{array}{c}\text { KGE } \\
\text { con act. } \\
\text { L (h) }\end{array}$ & $\begin{array}{c}\text { KGE } \\
\text { con act } \\
\text { L (h) }\end{array}$ \\
\hline & & & & & & & & 1 & 6 & 12 & 24 \\
\hline 4 & 882,3 & $\mid-0,903$ & 41,2 & 6,017 & 0,6701 & 0,9777 & 0,585 & 0,964 & 0,652 & 0,424 & 0,179 \\
\hline 22 & 482,1 & $-0,698$ & 444,5 & 5,909 & 0,9301 & 0,9457 & 0,897 & 0,942 & 0,932 & 0,890 & 0,802 \\
\hline IRSTEA 01 & 417,6 & $-1,375$ & 561,1 & 7,857 & & & & & & & \\
\hline IRSTEA 02 & 419,7 & $-0,762$ & 498,0 & 7,751 & & & & & & & \\
\hline
\end{tabular}

Se puede observar que el punto 22 está más próximo a los valores propuestos por el IRSTEA (que tiene una vasta experiencia en la operación del modelo de pronóstico en la cuenca en estudio), y se puede ver también que la performance del punto 4 se degrada rápidamente al mismo tiempo que el horizonte de pronóstico se prolonga. Esto quiere decir que los valores del punto 4 no son buenos más que para $\mathrm{L}=1 \mathrm{~h}$, pero el punto 22 es más robusto, como se muestra en la figura 7 .

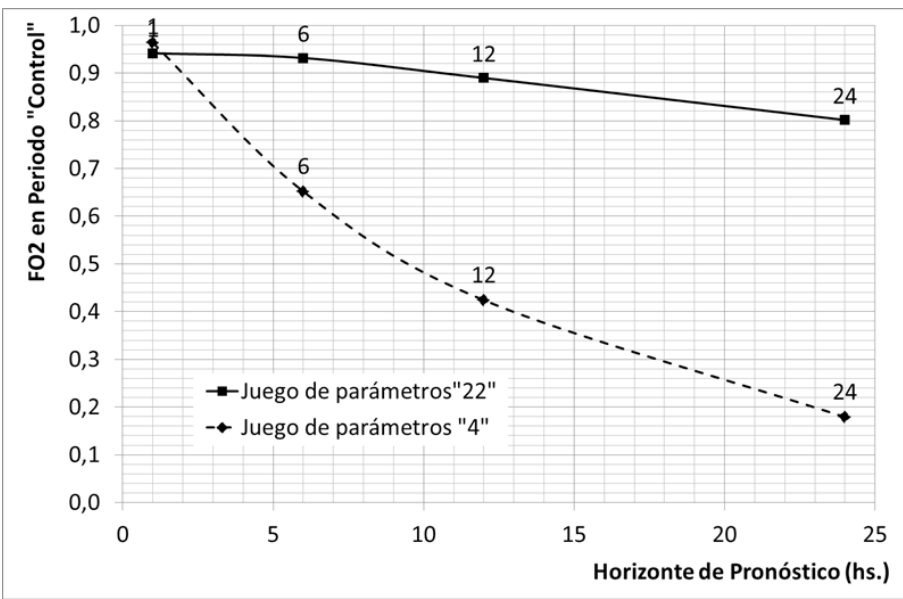

Figura 7. Sensibilidad de la performance (KGE) frente a variaciones en el horizonte pronosticado (L)

\section{RESULTADOS Y DISCUSIONES}

Teniendo en cuenta los resultados presentados, desde el punto de vista operacional se puede formular la siguiente pregunta: frente a la posibilidad de quedarse (en cualquier momento) sin los caudales observados durante el pronóstico (es decir, aquellos valores requeridos para realizar la actualización del estado del modelo), ¿cuál de los dos criterios siguientes será el más adecuado para adoptar?

Opción 1: Elegir de la población optimizada, el juego de parámetros que parece mejor, porque es bastante bueno para simular la salida del modelo en modo «con» y «sin» la actualización (por ejemplo el punto 22) y simular en cualquier escenario.

Opción 2: Elegir de la población optimizada el juego de parámetros con mejor performance en pronóstico (por ejemplo el punto 1), y frente a la eventual carencia de la información de caudal observado, cambiar al modo «sin la actualización», donde el modelo corre con el mejor juego de parámetros «sin la actualización» (por ejemplo el punto 25). 
Si existe algún punto capaz de representar todos los escenarios posibles con una performance aceptable, parece razonable adoptarlo como solución única (Opción 1), pero si la mejora de la performance en modo «sin la actualización», significa la degradación de la performance en modo «con la actualización» (o a la inversa), resulta de gran valor la disposición de las soluciones óptimas extremas (mejor FO1 y mejor FO2).

En consecuencia, para analizar cuál opción elegir en operación, eso dependerá en gran medida de la cuenca hidrográfica, ya que cada cuenca tiene su propia población óptima particular. En efecto, de la tabla 1 se puede extraer conclusiones únicamente para esa cuenca.

\section{CONCLUSIONES}

El modelo de pronóstico hidrológico GR4P es una poderosa herramienta para la estimación del caudal con un determinado horizonte de previsión. Sin embargo, la exactitud de la estimación es altamente dependiente de la disponibilidad a tiempo real del caudal observado, ya que le permite actualizar el estado del modelo. La carencia, durante la fase operativa, del caudal observado a tiempo real, produce que el modelo pase a funcionar como un simple modelo de simulación. El empleo de la optimización multi-objetivo para la calibración de la cuenca «Moros en Concarneau» entre ambos escenarios, permitió alcanzar un conjunto de soluciones con distintas performances en ambas FO.

Se observó que hay una notoria tendencia de desmejorar la performance debido a la actualización de estado, a medida que aumenta el horizonte L. Esto puede ser explicado porque el procedimiento de actualización del modelo, obliga a que a cada paso de tiempo el valor pronosticado tome exactamente el valor observado en cada Dt, quitándole de este modo grados de libertad en la selección del juego óptimo de parámetros.

Se puso en evidencia que la bondad de un juego de parámetros no sólo depende de la performance del modelo frente al horizonte de pronóstico de interés, sino también a la sensibilidad de la performance a variaciones en el tiempo de horizonte establecido, a medida que se aleja del horizonte con el cual fue calibrado.

Finalmente, se propone como herramienta operativa la optimización multi-objetivo de un modelo, a los fines de contar con mayor información de base frente a la decisión de adoptar uno o dos juegos de valores de parámetros capaces de adaptarse a los dos escenarios posibles durante operación: disponibilidad o no disponibilidad de los valores de cual observado a tiempo real.

\section{AGRADECIMIENTOS}

Se agradece al Programa STIC-AMSUD por el financiamiento del proyecto Hyradier, al Instituto Superior de Estudios Ambientales (ISEA) y al Centro de Tecnología del Agua (CETA), de la Universidad Nacional de Córdoba, Argentina.

\section{REFERENCIAS BIBLIOGRÁFICAS}

Berthet, L. 2010. Prevision des crues au pas de temps horaire: pour une meilleure assimilation de l'information de debit dans un modele bydrologique. These de doctorat, Cemagref / AgroParis'Tech, Paris.

Brazil, L.E., Krajewski, W.F. 1987. «Optimization of complex hydrologic models using random search methods». Paper presented at Conference on Engineering Hydrology, 
Hydraulics Division, American Society of Civil Engineering. Williamsburg, VA, 3-7 August.

Brazil, L.E. 1988. Multilevel calibration strategy for complex hydrologic simulation models. Ph.D. Dissertation, Colorado State University, Fort Collins, CO.

Duband, D. 1999. Réflexion actuelle sur la prévision bydrométéorologique. Colloque SHF «La gestion des risques liés aux inondations», 29 et 30 septembre 1999, Paris.

Duan, Q., Gupta, V.K., Sorooshian, S. 1992. Effective and efficient global optimization for conceptual rainfall-runoff models. Water Resources Research, 28 (4), 1015-1031.

Duan, Q., Gupta, V.K., Sorooshian, S. 1993. A shuffled complex evolution approach for effective and efficient global minimization. Journal of Optimization Theory and Applications, 76 (3), 501-521.

Gan, T.Y., Biftu, G.F. 1996. Automatic calibration of conceptual rainfall-runoff models: optimization algorithms, catchment conditions, and model structure. Water Resources Research, 32 (12), 3513-3524.

Gupta, H.V. y otros. 2009. Decomposition of the mean squared error and NSE performance criteria: Implications for improving hydrological modelling. Journal of Hydrology, Volume 377, Issues 1-2, 20 October 2009, Pages 80-91.

Klemeš, V. 1986. Operational testing of hydrological simulation models. Hydrological Sciences Journal, 31(1), 13-24.

Luce, C.H., Cundy, T.W. 1994. Parameter identification for a runoff model for forest roads. Water Resources Research, 30 (4), 1057-1069.

Oudin L., Michel, C., Anctil F. 2005. Which potential evapotranspiration input for a lumped rainfall-runoff model?. Part 1-Can rainfall-runoff models effectively handle detailed potential evapotranspiration inputs? Journal of Hydrology 303 (2005), 275-289.

Perrin, C., Michel, and V. Andréassian. 2003. Improvement of a parsimonious model for streamflow simulation. J. Hydrol. 279: 275-289.

Sorooshian, S.; Duan, Q. y Gupta, V.K. 1993. Calibration of rainfall-runoff models: application of global optimization to the Sacramento soil moisture accounting model. Water Resources Research, 29 (4), 1185-1194.

Tangara, M. 2005. Nouvelle méthode de prévision de crue utilisant un modèle pluie-débit global. Thèse de Doctorat, Cemagref / EPHE, Paris.

Tanakamaru, H. 1995. Parameter estimation for the tank model using global optimization. Transactions of the JSIDRE, 178, 103-112.

Tanakamaru, H. y Burges, S.J. 1996. Application of Global Optimization to Parameter Estimation of the Tank Model. In: Proceedings of the International Conference on. Water Resources and Environmental Research: Towards the 21th Century, Kyoto, Japan, 29-31 October 1996 (2), 39-46.

Wang, Q.J. 1991. The genetic algorithm and its application to calibrating conceptual rainfall-runoff models. Water Resources Research, 27 (9), 2467-2471.

Yapo, P.-O.; H. V. Gupta y S. Sorooshian. 1998. Multi-objective global optimization for bydrologic models. J. Hydrol. 204(1), 83-97.

Fecha de recepción: 25 de febrero de 2015

Fecha de aceptación: 30 de abril de 2015

\section{Correspondencia}

Facundo Alonso

facundojosealonso@gmail.com 